\title{
Exoskeleton transparency: feed-forward compensation vs. disturbance observer
}

\section{Journal Article}

\section{Author(s):}

Just, Fabian (1); Özen, Özhan; Bösch, Philipp; Bobrovsky, Hanna; Klamroth-Marganska, Verena (D); Riener, Robert; Rauter, Georg

Publication date:

2018-12

Permanent link:

https://doi.org/10.3929/ethz-b-000310187

Rights / license:

In Copyright - Non-Commercial Use Permitted

Originally published in:

at - Automatisierungstechnik 66(12), https://doi.org/10.1515/auto-2018-0069 


\title{
Applications
}

Fabian Just*, Özhan Özen, Philipp Bösch, Hanna Bobrovsky, Verena Klamroth-Marganska, Robert Riener and Georg Rauter

\section{Exoskeleton transparency: feed-forward compensation vs. disturbance observer}

\author{
Exoskelett Transparenz: Vorwärtssteuerung vs. Störbeobachter
}

https://doi.org/10.1515/auto-2018-0069

Received May 14, 2018; accepted October 25, 2018

Abstract: Undesired forces during human-robot interaction limit training effectiveness with rehabilitation robots. Thus, avoiding such undesired forces by improved mechanics, sensorics, kinematics, and controllers are the way to increase exoskeleton transparency.

In this paper, the arm therapy exoskeleton ARMin IV+ was used to compare the differences in transparency offered by using the previous feed-forward model-based controller, with a disturbance observer in a study. Systematic analysis of velocity-dependent effects of controller transparency in single- and multi-joint scenarios performed in this study highlight the advantage of using disturbance observers for obtaining consistent transparency behavior at different velocities in single-joint and multijoint movements. As the main result, the concept of the disturbance observer sets a new benchmark for ARMin transparency.

Keywords: exoskeleton, transparency, rehabilitation, physical human-robot interaction (pHRI)

Zusammenfassung: Ungewollte Kräfte bei MenschMaschine Interaktionen limitieren die Trainingsqualität mit Rehabilitationsrobotern. Mit dem Vermeiden dieser ungewollten Kräfte durch verbesserte Mechanik, Senso-

\footnotetext{
*Corresponding author: Fabian Just, Sensory-Motor Systems Lab, ETH Zurich and Spinal Cord Injury Center, University Hospital Balgrist, Zurich, Switzerland, e-mail: fabian.just@hest.ethz.ch Özhan Özen, ARTORG Center for Biomedical Engineering Research, Gerontechnology \& Rehabilitation, University of Bern, Bern, Switzerland, e-mail: oezhan.oezen@artorg.unibe.ch Philipp Bösch, Hanna Bobrovsky, Verena Klamroth-Marganska, Robert Riener, Sensory-Motor Systems Lab, ETH Zurich and Spinal Cord Injury Center, University Hospital Balgrist, Zurich, Switzerland, e-mails: phboesch@student.ethz.ch, hannab@student.ethz.ch, verena.klamroth@hest.ethz.ch, robert.riener@hest.ethz.ch Georg Rauter, Sensory-Motor Systems Lab, ETH Zurich and Spinal Cord Injury Center, University Hospital Balgrist, Zurich, Switzerland; and BIROMED-Lab, DBE, University of Basel, Basel, Switzerland, e-mail: georg.rauter@unibas.ch
}

rik, Kinematik und Regelungskonzepte wird die Transparenz des Exoskeletts erhöht. In diesem Beitrag wurde das Armexoskelett ARMin IV+ genutzt, um die zur Zeit verwendete modellbasierte Vorwärtssteuerung mit einem Störbeobachter in einer Studie zu vergleichen. Die systematische Analyse von geschwindigkeitsbasierten Effekten der Transparenz in Einzelgelenk- und Multigelenkszenarien hebt die Vorteile des Störbeobachters hervor, welcher konsistentes Transparenzverhalten bei verschiedenen Geschwindigkeiten während Einzelgelenk- und Multigelenksbewegungen zeigte. Als Hauptresultat setzt das Konzept des Störbeobachters einen neuen Maßstab für ARMin Transparenz.

Schlagwörter: Exoskelett, Transparenz, Rehabilitation, physikalische Mensch-Roboter Interaktion

\section{Introduction}

In rehabilitation, diagnosis, and therapy, robotic systems are commonly used for the treatment of upper extremity motor function impairments, with several studies showing that training with robots fosters motor recovery $[1,2,3]$. For transmitting the correct amount of supportive or resistive force to the patient and for the patient's inter-joint coordination, the underlying mechanical system needs to be mechanically transparent $[4,5,6]$. Transparency in physical human-robot interaction (pHRI) is defined in the literature in multiple, albeit similar ways: e. g., not to apply any assistance/resistance to free motion [5], or that the robot's reaction forces perceived by the user are minimal [7].

While quantitative evaluation of transparency in pHRI is performed through diverse assessments, no standard procedure exists $[4,8]$. In gait rehabilitation for example, the motor torques of the Lokomat were used to gain a rough estimate of robot transparency by calculation of the root mean square (RMS) interaction torques between the robot and the human [9]. Others directly measured the robothuman RMS interaction forces through force/torque sen- 
sors for several walking speeds by synchronizing walking cadence to a metronomic tone [10]. Improving upon these attempts, researchers on the FLOAT robot for gait rehabilitation even used a motorized test setup to assess transparency at different velocities and different levels of body weight support in a reproducible fashion. Here, the deviation from the desired interaction forces was assessed in all three directions using mean, standard deviation, and peak force at the pHRI interaction point during given trajectory movements [8]. In arm rehabilitation robotics, the transparency of the exoskeleton robot RehabExos was analyzed through subject's forces during a one degree of freedom movement, with the goal to minimize these physical human-robot interaction forces [11]. These forces were also measured through force/torque sensors attached to the robot. For the arm exoskeleton ABLE, a nine point performance index was developed combining multiple position, velocity, and tracking performance indices, joint range limitation indices and several force/torque performance indices [12]. These force/torque performance indices were also measured with force/torque sensors. Mean force/torque signals summarized over all sensors or splitted in the three coordinate system directions were analyzed on the sensor level [12]. Summarized, these papers in literature focus mainly on the direct evaluation of interaction force/torques on the sensor level. However, measurements in exoskeletal systems at the sensor level entail not only the force/torque needed to complete the movement, but also misalignment effects of human and robotic axes, passive tissue deformation forces/torques and partial arm unloading effects on the exoskeleton that all can be independent of the evaluation of the particular movement or movement axes. Transforming the force/torque information from the sensor level on the robotic axes through the corresponding Jacobian orders influences axes depending, leading to a more neutral transparency analysis, especially for systems with several sensors that have different lever arms and therefore, different impact on the robotics axes. Nevertheless, transparency analysis on the axis (joint) level is rarely done in literature [13]. A systematic analysis on the joint level is needed to evaluate behavior and performance of transparency controllers. Since transparency performance of different dynamic movements should be equally good, the evaluation should concentrate on the effects of different movement velocities. As an additional measure to mean pHRI torques also joint peak torques should be analyzed. Only rarely joint peak torques were the focus of the transparency analysis [13], although they are directly related to the stick/slip effect that is one of the most prominent disturbance components opposing robot transparency [13, 14].

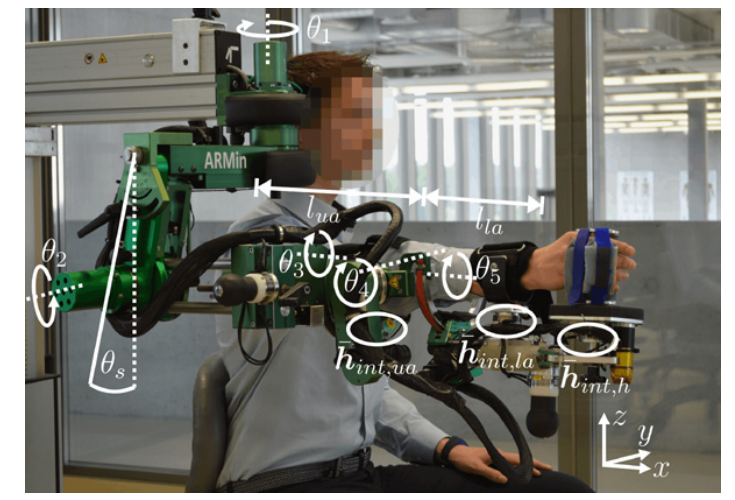

Figure 1: The ARMin IV+ exoskeleton robot with the three physical human-robot interaction (pHRI) wrenches $\overline{\boldsymbol{h}}_{\text {int, } S_{i}}$ from force/torque sensors $S_{i}$ at upper arm (ua), lower arm (la), and hand (h) are circled. The shoulder angle, $\theta_{s}$, the robot axes (joints) $\boldsymbol{\theta}$, and arm lengths $l_{u a}$, and $l_{l a}$ are marked respectively. At this position all three sensors local coordinate systems are in the indicated global coordinate system (Axis $x$ is parallel to the sagittal axis).

This paper focuses on the assessment of the transparency of the exoskeleton robot ARMin IV+. ARMin IV+ is an actuated seven degrees of freedom (DoFs) exoskeleton robot and has a six DoFs force/torque sensor at all three interaction points between human and robot at the upper arm (ua), the lower arm (la), and the hand (h) (see Fig. 1). Based on the measured force/wrench at the pHRI points through the three sensors, we want to evaluate transparency systematically through mean and peak pHRI forces and torques. Such a systematic evaluation allows quantifying the performance of the robot and enables comparing performance of different control strategies in a standardized way. We also introduce a generalizable evaluation metric, which is used to assess the transparency of each single axis (joint). As a generalization, the evaluation metric is expanded to multi-axis evaluation to consequently assess transfer of transparency characteristics from single-joint to multi-joint evaluation. All assessments were performed at two different velocities. As a systematic analysis, we evaluate the current model-based controller of ARMin IV+ and compare its results to a newly implemented disturbance observer, which is already used in motion control systems previously described in literature [15].

Section 2 summarizes the applied methods including an analysis of transparency metrics, the ARMin IV+ robot with its Jacobian, the two control concepts, and the study designs. Next, section 3 presents the results of the two studies and the questionnaire used. Subsequently, section 4 discusses the results of quantitative and subjective data, and finally, in section 5 conclusions on the results of the paper are drawn. 


\section{Methods}

In this section, the technical term "transparency" is defined. Consequently, the equations that will be needed for assessment of transparency, different evaluation concepts, and their implications will be derived. In the following, the application of these equations on the ARMin rehabilitation robot are shown and the ARMin Jacobian information is provided. Subsequently, the two investigated controllers, i) a feed-forward model-based controller and ii) a disturbance observer are presented. Finally, an overview on subjects and ethics is given before introducing the designs for a single-joint and a multi-joint transparency study and their primary outcomes measures.

\subsection{Implications of the transparency definition}

All variations of systems' transparency definitions in literature aim to reach a full disturbance compensation [5, $7,16]$. Optimally, the estimated disturbances $\hat{\boldsymbol{\tau}}_{\text {dis }}$ can fully compensate for the effective disturbances $\boldsymbol{\tau}_{\text {dis }}$ of a system:

$$
\text { System transparency definition: } \boldsymbol{\tau}_{\text {dis }}-\hat{\boldsymbol{\tau}}_{\text {dis }}=\mathbf{0} \text {. }
$$

In reality however, a disturbance will never be fully compensated e. g., due to noise, communication delay, inertia, and limited update rate. Even in an physical human-robot interaction system perfectly compensating the mentioned disturbances, there will always remain a minimum of disturbance $f\left(\boldsymbol{\tau}_{\text {dis }}\right)$ for two reasons. First, robotic systems are causal, i. e., the disturbance compensation can only be applied after the disturbance has been measured. Second, the human user creates interactions $\boldsymbol{h}_{\text {int }}$ that depend on the actual pose of the robot, the desired torques of the currently selected robotic training scenario $\boldsymbol{\tau}_{d e s}$, the perceived disturbances, and the human's movement capabilities and intention. However, the resulting interactions that are initiated by the human user $\boldsymbol{h}_{\text {int }}$ with the system cannot be known a priori. Therefore, the controller has to wait until interactions can be measured $\overline{\boldsymbol{h}}_{\text {int, } S_{i}}$ e. g., by a force/torque sensor $S_{i}$. The measured six dimensional force-torque interactions are called interaction wrench $\overline{\boldsymbol{h}}_{\text {int }, S_{i}}$. For the application of the transparency objective in (1), the interaction wrench $\boldsymbol{h}_{\text {int }}$ needs to be analyzed. In literature, mainly the direct wrench signals at the single pHRI points $\overline{\boldsymbol{h}}_{\text {int }, S_{i}}$ were in the focus of evaluation [12, 10, 11]. For multidimensional rehabilitation robots like ARMin lever arms from both sides of an axis exist. Transparency evaluation isolated only on the sensor level $\overline{\boldsymbol{h}}_{\text {int, } S_{i}}$ entails not only the disturbances in movement direction, but also all other force/torque disturbances in all coordinate system directions. These other force/torque disturbances are misalignment effects of the human axes to the robotic axes, muscle tension of the human, skin deformation effects as well as unloading effects of the arm on the robotic structure, especially when the freedom of movement of the arm is limited against gravity. These other disturbances can be independent of the force/torque needed to exert the particular evaluated movement, but they still fully influence the transparency evaluation on the sensor level.

However, another way of analyzing human-robot interactions is to transform the measured interaction wrench $\overline{\boldsymbol{h}}_{i n t, S_{i}}$ at sensor $S_{i}$ to pHRI torques on the robot axes $\boldsymbol{\tau}_{S_{i}}$ by means of the corresponding Jacobian $\boldsymbol{J}_{\text {int, } S_{i}}$ [13]. This transformation on the axes level allows to analyze the effect of the disturbances on each axis separately leading to a more neutral transparency analysis. Especially if multiple pHRI interaction points exist with different lever arms on one robot axis, then the proportional influence of each measured interaction wrench $\overline{\boldsymbol{h}}_{i n t, S_{i}}$ on the robotic axis can only be determined through the corresponding Jacobian on the axes level and not on the sensor level. At the same time, the pHRI torques $\boldsymbol{\tau}_{S_{i}}$ are anyways required as an input for the majority of controllers (e.g., admittance control). All measured interaction wrenches $\overline{\boldsymbol{h}}_{i n t, S_{i}}$ mapped on all robot axes $\boldsymbol{\tau}_{S_{i}}$ can be summed up to the pHRI torque at the corresponding axes (joints) $\boldsymbol{\tau}_{\mathrm{pHRI}}$. The summarized torque vector $\boldsymbol{\tau}_{\text {pHRI }}$ for all five robot axes induced by all $N$ pHRI wrenches $\overline{\boldsymbol{h}}_{\text {int }, S_{i}}$ can be calculated

$$
\boldsymbol{\tau}_{\mathrm{pHRI}}=\sum_{i=1}^{N} \boldsymbol{J}_{i n t, S_{i}}^{T} \cdot \overline{\boldsymbol{h}}_{i n t, S_{i}}=\sum_{i=1}^{N} \boldsymbol{\tau}_{S_{i}},
$$

where $\boldsymbol{J}_{i n t, S_{i}}^{T}$ represents the Jacobian of sensor $S_{i}$ in the sensor frame mapping to the robot joints and the measured wrench $\overline{\boldsymbol{h}}_{i n t, S_{i}}$ in sensor frame, embodies the corresponding force/torque sensor data. The product of Jacobian and force/torque sensor data, $\boldsymbol{\tau}_{S_{i}}$ is the applied torque on all robot axes caused by the pHRI sensor $S_{i}$. The general equation (2) can be specified, since ARMin IV+ is equipped with three $(N=3)$ pHRI points $S_{i} \in[u a, l a, h]$ :

$$
\begin{aligned}
& \boldsymbol{\tau}_{\mathrm{pHRI}}=\boldsymbol{J}_{i n t, u a}^{T} \cdot \overline{\boldsymbol{h}}_{i n t, u a}+\boldsymbol{J}_{i n t, l a}^{T} \cdot \overline{\boldsymbol{h}}_{i n t, l a}+\boldsymbol{J}_{i n t, h}^{T} \cdot \overline{\boldsymbol{h}}_{i n t, h} \\
& \boldsymbol{\tau}_{\mathrm{pHRI}}=\boldsymbol{\tau}_{u a}+\boldsymbol{\tau}_{l a}+\boldsymbol{\tau}_{h},
\end{aligned}
$$

where exemplary the Jacobian $\boldsymbol{J}_{\text {int, ua }}^{T}$ has dimension [5x6], the interaction wrench $\boldsymbol{h}_{\text {int,ua }}$ has dimension [6x1], and the $\boldsymbol{\tau}_{u a}$ has dimension [5x1] for the following five axes evaluation of ARMin IV+. 


\subsection{ARMin IV+ exoskeleton robot}

The ARMin IV+ exoskeleton robot [17] has seven actuated DoFs

$$
\boldsymbol{\theta}=\left[\theta_{1}, \theta_{2}, \theta_{3}, \theta_{4}, \theta_{5}, \theta_{6}, \theta_{7}\right]^{T}
$$

$\theta_{1}$ (horizontal shoulder abduction/adduction), $\theta_{2}$ (shoulder elevation), $\theta_{3}$ (internal /external shoulder rotation), $\theta_{4}$ (elbow flexion/extension), $\theta_{5}$ (forearm pronation/supination), $\theta_{6}$ (wrist flexion/extension), and $\theta_{7}$ (hand opening/closing). In this paper, the first five axes are evaluated. Axis $\theta_{6}=0$ (neutral flexion) and $\theta_{7}=0$ (closed hand) are position controlled by a PD controller. Therefore, for simplicity reasons the vectors for torque and position in this paper contain the first five ARMin axes, and the vector sizes are [5x1]. For calculating the robot's kinematics and inertia matrices, potentiometers measure all possible anthropometric exoskeleton adaptations to the user's body in absolute values. This includes online measurements of the upper arm length $l_{u a}$, the lower arm length $l_{l a}$, and the shoulder angle $\theta_{s}$ of the ARMin IV+ exoskeleton (see Fig. 1). This small but important expansion of the ARMin's sensor concept allows including major mechanic adaptations in the feed-forward control of the device [17].

Table 1: Influence of the components of the three force/torque sensors $S_{u a}, S_{l a}$, and $S_{h}$ in sensor coordinate system on the robot axes torque $\left(\tau_{1}, \ldots, \tau_{5}\right)$ through the partially decoupled Jacobian. " $x$ " and " 0 " represent "influence" or "no influence" on the axis torque through the sensor element across its range of motion during the study. The concordant coordinate system for all three sensors is displayed in Fig. 1.

\begin{tabular}{lcccccc}
\hline $\boldsymbol{\tau}_{S_{u a}}$ & $F_{x}$ & $F_{y}$ & $F_{z}$ & $\tau_{x}$ & $\tau_{y}$ & $\tau_{z}$ \\
\hline$\tau_{1}$ & 0 & $\mathrm{x}$ & $\mathrm{x}$ & $\mathrm{x}$ & $\mathrm{x}$ & $\mathrm{x}$ \\
$\tau_{2}$ & 0 & $\mathrm{x}$ & $\mathrm{x}$ & 0 & $\mathrm{x}$ & $\mathrm{x}$ \\
$\tau_{3}$ & 0 & 0 & 0 & $\mathrm{x}$ & 0 & 0 \\
$\tau_{4}$ & 0 & 0 & 0 & 0 & 0 & 0 \\
$\tau_{5}$ & 0 & 0 & 0 & 0 & 0 & 0 \\
\hline \hline $\boldsymbol{\tau}_{S_{l a}}$ & $F_{x}$ & $F_{y}$ & $F_{z}$ & $\tau_{x}$ & $\tau_{y}$ & $\tau_{z}$ \\
\hline$\tau_{1}$ & $\mathrm{x}$ & $\mathrm{x}$ & $\mathrm{x}$ & $\mathrm{x}$ & $\mathrm{x}$ & $\mathrm{x}$ \\
$\tau_{2}$ & $\mathrm{x}$ & $\mathrm{x}$ & $\mathrm{x}$ & $\mathrm{x}$ & $\mathrm{x}$ & 0 \\
$\tau_{3}$ & 0 & $\mathrm{x}$ & $\mathrm{x}$ & 0 & $\mathrm{x}$ & $\mathrm{x}$ \\
$\tau_{4}$ & 0 & $\mathrm{x}$ & $\mathrm{x}$ & 0 & $\mathrm{x}$ & $\mathrm{x}$ \\
$\tau_{5}$ & 0 & 0 & 0 & $\mathrm{x}$ & 0 & 0 \\
\hline \hline $\boldsymbol{\tau}_{S_{h}}$ & $F_{x}$ & $F_{\mathrm{y}}$ & $F_{z}$ & $\tau_{x}$ & $\tau_{y}$ & $\tau_{z}$ \\
\hline$\tau_{1}$ & $\mathrm{x}$ & $\mathrm{x}$ & $\mathrm{x}$ & $\mathrm{x}$ & $\mathrm{x}$ & $\mathrm{x}$ \\
$\tau_{2}$ & $\mathrm{x}$ & $\mathrm{x}$ & $\mathrm{x}$ & $\mathrm{x}$ & $\mathrm{x}$ & $\mathrm{x}$ \\
$\tau_{3}$ & 0 & $\mathrm{x}$ & $\mathrm{x}$ & $\mathrm{x}$ & $\mathrm{x}$ & $\mathrm{x}$ \\
$\tau_{4}$ & 0 & $\mathrm{x}$ & $\mathrm{x}$ & 0 & $\mathrm{x}$ & $\mathrm{x}$ \\
$\tau_{5}$ & 0 & 0 & 0 & $\mathrm{x}$ & 0 & 0 \\
\hline
\end{tabular}

Three 6 DoFs force/torque sensors measure the pHRI at all three interaction points upper arm (ua), lower arm (la), and hand (h). Due to the placement of interaction points, sensor (ua) measures influences on the first three axes, sensor (la), on the first five axis, and sensor (h), on the first six axes. The Jacobian that is applied to distribute the measured pHRI interaction wrench at the sensors to torques at the joints is partially decoupled to allow the human subjects for more intuitive multi-joint movements (see Tab. 1). The decoupling focuses on the upper arm rotation (axis 3), which is only controlled by torques of sensor $S_{u a}$ not with forces of sensor $S_{u a}$. Similarly, the lower arm rotation (axis 5) is only torque controlled by sensors $S_{l a}$ and $S_{h}$. Importantly, a rotation of the lower arm induced through torque $\tau_{x}$ at the lower arm sensor $S_{l a}$ does not create a rotation of the upper arm (see Tab. 1). A rotation induced by $\tau_{x}$ of the hand sensor $S_{h}$ instead is leading to upper and lower arm rotation. This particular chosen decoupling of upper and lower arm rotation should enable more intuitive multi-joint movements due to an increase in similarity to single-joint movement control in ARMin.

During movement the major disturbances that influence the performance of the ARMin exoskeleton robot are: gravitation, friction, inertia, cable and spring elasticity. For robot torque characteristics in the Lagrangian form the following sum of disturbances can be defined:

$$
\boldsymbol{\tau}_{d i s}=\boldsymbol{M}(\boldsymbol{\theta}) \ddot{\boldsymbol{\theta}}+\mathbf{g}_{r}(\boldsymbol{\theta})+\boldsymbol{\eta}_{e}(\boldsymbol{\theta}, \dot{\boldsymbol{\theta}}),
$$

where $\boldsymbol{M}$ represents the mass matrix of the actuated exoskeleton. The function $\mathbf{g}_{r}$ contains the gravitational effects of the robot. The symbol, $\boldsymbol{\eta}_{e}$, summarizes all position, velocity and acceleration-dependent effects like spring and cable elasticity, friction effects as well as all other effects that can not be modeled. Fig. 2 shows the

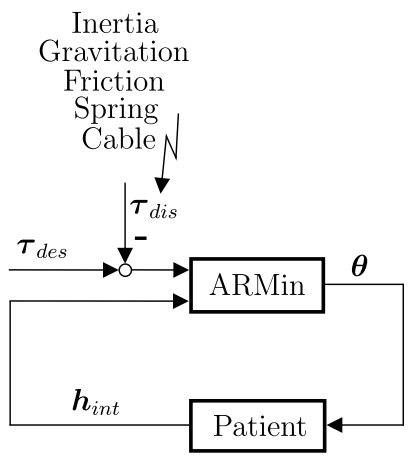

Figure 2: Mechanical system of plant ARMin IV+ and patient connected through the actual joint positions $\boldsymbol{\theta}$ and the generalized patient interaction wrenches $\boldsymbol{h}_{\text {int }}$, which is representative for all three pHRI points at the upper arm, lower arm, and hand. Furthermore, the disturbance torques $\boldsymbol{\tau}_{d i s}$ and desired torques $\boldsymbol{\tau}_{\text {des }}$ are inputs to the plant ARMin. 
disturbances $\boldsymbol{\tau}_{\text {dis }}$ in the control loop as well as the influences of the generalized patient interaction wrench $\boldsymbol{h}_{\text {int }}$, which includes data from all three pHRI points of ARMin. Safety of the human in ARMin during operation with different controllers is tackled through a safety system incorporating limitations for the end-effector workspace, joint workspace, velocities and wrench on the joint and endeffector level.

\subsection{Feed-forward model-based controller}

A model-based controller uses physical feed-forward models to compensate unwanted disturbances $\boldsymbol{\tau}_{d i s}$, which are usually subsystems or physical properties of the exoskeleton [11]. The feed-forward model consists of several modelbased controllers that allow online adaptive compensation (OAC). Hereby, the OAC refers to the online adaption of ARMin's geometry to the anthropometry of the patient [17]. The OAC expands the model-based controller that has been implemented and successfully tested in a large interventional study [1]. The OAC is currently used in our ARMin IV+ devices in the clinics.

As seen in Fig. 3, independent models for compensating cable and spring elasticity as well as friction and gravitation are implemented. All models are provided with real-time position data of each axis by position sensors. In the friction model, the position data is differentiated

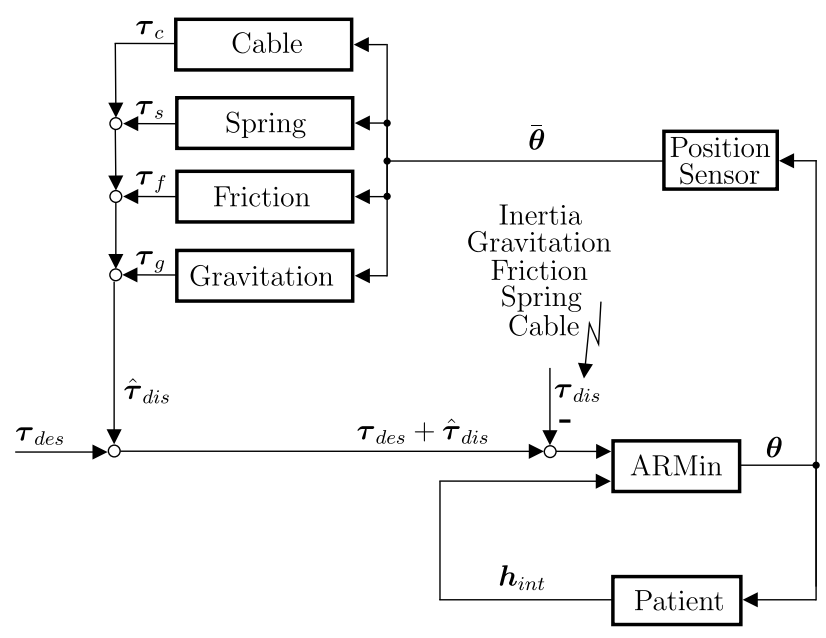

Figure 3: The feed-forward model-based controller includes models for gravitation, friction, spring, and cable elasticity [17]. Summarizing all model torques, the disturbances estimation $\hat{\boldsymbol{\tau}}_{\text {dis }}$ should compensate the disturbances $\boldsymbol{\tau}_{\text {dis }}$ of the exoskeleton ARMin. The signals $\hat{\boldsymbol{\tau}}_{\text {dis }}$ and $\boldsymbol{\tau}_{\text {des }}$ are the input to the plant ARMin. The patient-robot interaction wrench $\boldsymbol{h}_{\text {int }}$ is representative for all three interaction points at the upper arm, lower arm and hand. once to receive additional velocity information. An inertia compensation model was not implemented, because of high delays to derive an accurate acceleration signal through two times differentiation of the encoder position information [18]. The missing inertial compensation is expected to create higher measured mean and peak torques of $\boldsymbol{\tau}_{\mathrm{pHRI}}$, nevertheless dynamic movements in ARMin are not restricted thereby [18]. Each model is described in detail in [17]. All model-based controllers provide desired torque output to ARMin at $1000 \mathrm{~Hz}$ to compensate the disturbances with an estimation $\hat{\boldsymbol{\tau}}_{\text {dis }}$ in a feed-forward fashion (see Fig. 3). The axis-wise sums of the model torques are consequently applied to ARMin to compensate the exoskeleton's disturbance $\boldsymbol{\tau}_{\text {dis }}$ :

$$
\hat{\boldsymbol{\tau}}_{d i s}=\boldsymbol{\tau}_{c}+\boldsymbol{\tau}_{s}+\boldsymbol{\tau}_{f}+\boldsymbol{\tau}_{g} .
$$

\subsection{Velocity-based disturbance observer}

A velocity-based disturbance observer is a robust closedloop acceleration controller using velocity and inertia information of the ARMin. The observer controls the inertial parameters of each axis individually with a virtual nominal axis inertia $m_{n}$. The concept for the velocity-based disturbance observer from [15] was implemented in the ARMin IV+ for 5 DoFs. The controller converts the mass matrix $\boldsymbol{M}(\boldsymbol{\theta})$ as introduced in (5) into the nominal mass matrix $\boldsymbol{M}_{\text {nom }}$ containing only virtual nominal inertia entries $m_{n}$ on the main diagonal for all five axes

$$
\boldsymbol{M}_{\text {nom }}=\operatorname{diag}\left(m_{n, 1}, m_{n, 2}, m_{n, 3}, m_{n, 4}, m_{n, 5}\right) .
$$

The disturbance observer is changing the plant, ARMin (see Fig.4), into a double integrator with the defined virtual nominal inertia parameters $m_{n}$ of (7). Since the controller is implemented as a single axis controller, no further axes indexing is required. The measured position data $\overline{\boldsymbol{\theta}}$ as shown in Fig. 4 is used for the derivation. The inertia $m(\overline{\boldsymbol{\theta}})$ of an arbitrary axis abstracted from the inertia matrix $\boldsymbol{M}(\overline{\boldsymbol{\theta}})$,

$$
m(\overline{\boldsymbol{\theta}})=m_{n}+\Delta m(\overline{\boldsymbol{\theta}}),
$$

is always the sum of its axis corresponding nominal inertia $m_{n}$ and remaining inertia $\Delta m(\overline{\boldsymbol{\theta}})$. The remaining inertia $\Delta m(\overline{\boldsymbol{\theta}})$ is treated as an disturbance and thus compensated for through the disturbance observer. Importantly, since the remaining inertia $\Delta m(\overline{\boldsymbol{\theta}})$ entails also all non-diagonal entries of the mass matrix, the compensation of these lead to an enhanced axes decoupling. The single axis definition of disturbance therefore yields

$$
\tilde{\tau}_{d i s}=\Delta m(\overline{\boldsymbol{\theta}}) \ddot{\bar{\theta}}+g_{r}(\overline{\boldsymbol{\theta}})+\eta_{e}(\overline{\boldsymbol{\theta}}, \dot{\overline{\boldsymbol{\theta}}}),
$$




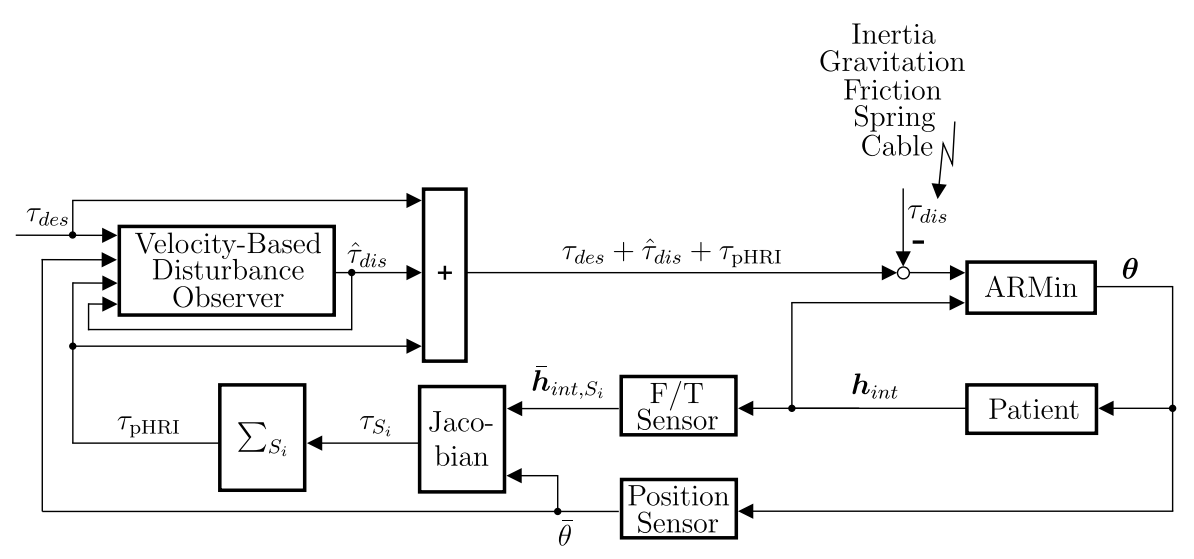

Figure 4: Single axis control loop with the velocity-based disturbance observer. The patient-robot interaction wrench $\boldsymbol{h}_{\text {int }}$ is measured at each interaction point $S_{i}$ with force/torque sensors as $\overline{\boldsymbol{h}}_{i n t, S_{i}}$. Multiplied with the respective Jacobian using all position sensor information $\bar{\theta}$, the robot axes torque $\tau_{S_{i}}$ is calculated. Summarizing all $\tau_{S_{i}}$ of all sensors, as seen in (2), results in $\tau_{\mathrm{pHRI}}$. The sum $\tau_{\text {des }}+\hat{\tau}_{\text {dis }}+\tau_{\mathrm{pHRI}}$ is the control input for the plant ARMin.

where $g_{r}(\overline{\boldsymbol{\theta}})$ and $\eta_{e}(\overline{\boldsymbol{\theta}}, \dot{\overline{\boldsymbol{\theta}}})$ are the scalar axis entries of the corresponding vectors of (5) and $\tilde{\tau}_{d i s}$ is an approximation of the unknown disturbance $\tau_{d i s}$.

As seen in Fig. 4, one input to the velocity-based disturbance observer is the vector of pHRI on the axes level $\boldsymbol{\tau}_{\mathrm{pHRI}}$ that encodes the movement intention of the patient from all robot-patient interaction points: upper arm cuff, lower arm cuff, and hand cuff. Another input to the velocity-based disturbance observer is the desired torque from higher level control, $\tau_{d e s}$, which summarizes all game and safety-related torques that shape the haptic environment of the exoskeleton. Without higher level control $\left(\tau_{\text {des }}=0\right)$, only input from the patient is taken into account. The sum of all input torques reduced by the actual inertial torque of the axis is an applied and complementary definition of the approximated disturbance presented in (9) through the sum of all disturbances and can be seen as equivalent.

$$
\tilde{\tau}_{\text {dis }}=\tau_{\text {des }}+\tau_{\mathrm{pHRI}}-m_{n} \ddot{\bar{\theta}},
$$

because $\tilde{\tau}_{\text {dis }}=0$ when the summed input torque is equal to what is currently executed on the axis. However, since acquiring real-time axis acceleration information is very costly and may be not feasible for only axis position sensing systems like ARMin, further assumptions of the velocity-based disturbance observer are taken to estimate (10).

During derivation of the velocity-based disturbance observer theory, it follows that for quasi-static approximated disturbances,

$$
\dot{\tilde{\tau}}_{\text {dis }}=0 \text {, }
$$

the estimation error decays to zero. However, most disturbances are not quasi-static leading to non-zero error for the disturbance estimation $\hat{\tau}_{\text {dis }}$. This limits performance with respect to robot transparency across different velocities. Therefore, the limitation introduces a systematic error behavior that will increase differences between the approximated $\tilde{\tau}_{\text {dis }}$ and real disturbance $\tau_{\text {dis }}$ with increasing joint velocities. Through this control design limitation it follows that the estimated disturbance $\hat{\tau}_{\text {dis }}$ is equal to the approximated disturbance $\tilde{\tau}_{\text {dis }}$ from (10) delayed by the following low-pass filter represented in the Laplace s-domain:

$$
\hat{\tau}_{d i s}=\frac{\omega_{c}}{s+\omega_{c}} \tilde{\tau}_{d i s}
$$

where $\omega_{c}$ is the low-pass filter angular cut-off frequency. Since the approximated disturbance $\tilde{\tau}_{d i s}$ is not known, the derivation continued with (10) in combination with the velocity limitation leading to (12). Finally, the velocitybased disturbance observer estimates the disturbance from (10) through the summarized and low-pass filtered input torque.

$$
\hat{\tau}_{d i s}=\frac{\omega_{c}}{s+\omega_{c}}\left(\hat{\tau}_{d i s}+\tau_{d e s}+\tau_{\mathrm{pHRI}}+\dot{\bar{\theta}} m_{n} \omega_{c}\right)-\dot{\bar{\theta}} m_{n} \omega_{c}
$$

Due to the low computational effort of the disturbance observer, the sampling rate of ARMin IV+ could be increased to $1800 \mathrm{~Hz}$. For the five axes that were controlled by the disturbance observer, the chosen low-pass filter cutoff frequencies that also determine the bandwidth of the controller and consecutively the bandwidth of ARMin are tuned in a region above the clinically relevant movement speed of human arms:

$$
\begin{aligned}
\boldsymbol{\omega}_{c} & =2 \pi \boldsymbol{f}_{c} \\
\boldsymbol{f}_{c} & =[10 \mathrm{~Hz}, 16 \mathrm{~Hz}, 30 \mathrm{~Hz}, 48 \mathrm{~Hz}, 110 \mathrm{~Hz}] .
\end{aligned}
$$


For systems like ARMin where only joint position information is available, joint acceleration information through differentiation or additional hardware is costly. Therefore, the velocity-based disturbance observer taking velocity information could be an alternative giving a compromise between performance and delay.

\subsection{Subjects and ethics}

All studies and the questionnaire were performed with 20 healthy subjects (10 females and 10 males, 19 righthanded, mean age: $26.2 \pm 2.2$ years; mean height: $1.75 \pm 0.09 \mathrm{~m}$; mean weight: $67.7 \pm 11.1 \mathrm{~kg}$ ). All studies were approved by the responsible institutions (KEKZH-Nr. 2015-0013, Zurich, Switzerland, and on clinicaltrials.gov NCT02720341).

\subsection{Single-joint transparency study}

In a single-joint transparency study, the transparency of the first five axes of the ARMin were evaluated individually with $\boldsymbol{\tau}_{\text {pHRI }}$ on both controllers to investigate transparency for each single axis across velocities. The subject was guided towards a predefined starting position by a position controller. Only the axis that had to be evaluated in the respective condition was set to transparent mode $\left(\tau_{\text {des }}=0\right)$ while all other axes remained in fixed PD position control-mode to eliminate the movement influence of other DoFs. A screen was placed in front of the subject that showed a moving reference object (red car) and a marker corresponding to the current axis position (blue car) as seen in Fig. 5 a). The subject was asked to follow the red reference car with the own blue car over the range of motion of the axis in transparent mode at constant reference speed (see Tab.2). Successful tracking of the red
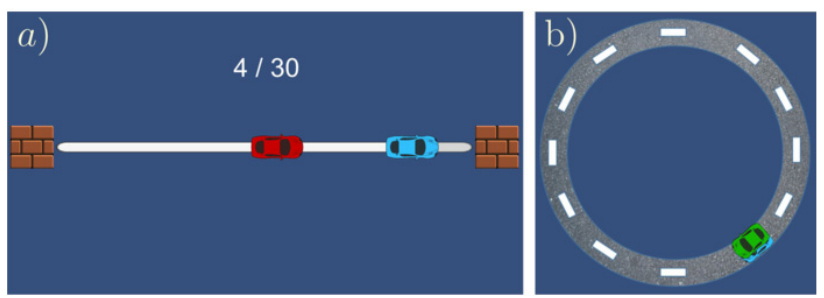

Figure 5: Visual feedback for the a) Single-Joint Transparency Study, b) Multi-Joint Transparency Study. The reference object is the red car that has to be tracked with the motion of the respective axis mapped to the position of the blue car. To indicate that the blue car is close enough to the red reference car, the blue car turns its color into green.
Table 2: Axes range of motion and corresponding "slow" and "fast" angular speeds of the single-joints for the transparency study. Anatomical axes were defined according to [19].

\begin{tabular}{rrrr}
\hline Axis & Range of Motion $^{\circ}$ & Slow $_{\overline{\bar{s}}}^{\circ}$ & Fast $^{\circ} \overline{\boldsymbol{s}}$ \\
\hline 1 & $(15,90)$ & 15 & 30 \\
2 & $(40,80)$ & 10 & 20 \\
3 & $(0,65)$ & 20 & 40 \\
4 & $(15,90)$ & 20 & 40 \\
5 & $(-65,65)$ & 45 & 90 \\
\hline
\end{tabular}

car was indicated by the red reference car color turning to green (see Fig. 5). Additional auditory feedback in form of a metronome sound was provided to help the subject maintain the desired movement pace. In each condition, 30 repetitions across the full range of motion were recorded. The condition was finished after 30 repetitions.

For each axis a "slow" and a "fast" condition were tested. The tested speeds and ranges of motion for each axis in Tab. 2 were chosen to cover the upper and lower limits of velocity and motion range from experience of daily usage of ARMin in clinics according to therapists respectively.

The order of the model-based controller and the disturbance observer was randomized for each subject. In each controller block, the order of axes tested was randomized for each subject. For each axis, first the "slow" scenario, and then the "fast" scenario was executed.

The corresponding axis torque signal $\tau_{\mathrm{pHRI}}$ from the full recorded vector $\boldsymbol{\tau}_{\mathrm{pHRI}}$ in (3) shows the influence of all force/torque sensors in and against the axis movement direction. Therefore, the axis torque signal $\tau_{\mathrm{pHRI}}$ is taken for the evaluation of the remaining axis torque. Similar to the interpretation of different transparency evaluation outcomes used in literature, the absolute mean [12] and absolute peak torque [8] are analyzed in this paper. The first primary outcome measure was the mean absolute humanrobot interaction torque in the corresponding movement axis direction

$$
\tau_{\mathrm{m}, \mathrm{pHRI}}=\frac{1}{n} \sum_{k=1}^{n} \operatorname{mean}\left|\boldsymbol{\tau}_{\mathrm{pHRI}, k}\right|,
$$

where $\boldsymbol{\tau}_{\mathrm{pHRI}, k}$ is the vector with all measurements of movement repetition $k$ and $n=30$ is the number of movement repetitions. This measure provides valuable information about the average interaction torque felt in and against movement direction. The second primary outcome measure is the mean peak absolute human-robot interaction 
torque in the direction of movement over all repetitions.

$$
\tau_{\mathrm{p}, \mathrm{pHRI}}=\frac{1}{n} \sum_{k=1}^{n} \max \left|\boldsymbol{\tau}_{\mathrm{pHRI}, k}\right|
$$

Through the importance of mean peak analysis, as discussed in the introduction, this outcome measure provides an impression about the maximum torque felt in and against movement direction.

\subsection{Multi-joint transparency study}

Directly after the single-joint transparency study, a multijoint transparency study with the respective controller was executed. Since the application field of the seven DoFs exoskeleton robot ARMin is mainly multi-joint movements and to assess transfer characteristics of the two controllers from single-joint evaluation to multi-joint evaluation, we decided to also evaluate transparency of the two controllers for multi-joint movements. Therefore, the participants were asked to track a reference object on a circular path with the end-effector parallel to the coronal plane in front of them (see Fig. 5 b). All five relevant axes (joints) $\left(\theta_{1}, \ldots, \theta_{5}\right)$ were in transparent mode $\left(\tau_{\text {des }}=0\right)$, the wrist was fixed in neutral flexion $\left(\theta_{6}=0\right)$ and the hand was fixed in a closed position $\left(\theta_{7}=0\right)$. The diameter of the circle was $d_{c}=0.28 \mathrm{~m}$ and the center position was set to the subject's chest height to cover typical activities of daily living like grasping and reaching tasks like in a repetitive rehabilitation training. 10 repetitions each for a "slow" $\left(45_{\frac{-}{s}}^{\circ}\right)$ and "fast" movement speed $\left(90_{\bar{s}}^{\circ}\right)$ were tested for each controller and participant.

Primary outcome measures were similar to the singlejoint transparency study: $\tau_{\mathrm{m}, \mathrm{pHRI}}$, the mean absolute pHRI torque, and $\tau_{\mathrm{p}, \mathrm{pHRI}}$, mean peak absolute pHRI torque in the direction of movement of all five movement axes. This time, the number of movement repetitions was $n=10$ instead of 30 (see equations (15), (16)). Single-joint and multi-joint transparency studies were performed on the same cohort and as part of the same experiment. Therefore, the order of the controllers was blockwise randomized for each subject.

\subsection{Questionnaire}

A questionnaire was issued to the participants to evaluate the subjective performance of the two controllers. After performing the single-joint and multi-joint transparency study, but before switching controllers, the following statements were assessed:
1. I felt disturbances during my movements.

2. Exact movements were difficult to perform.

The participants could rate each statement on a 7-point Likert scale (strongly agree, agree, slightly agree, uncertain, slightly disagree, disagree, strongly disagree). After the single-joint and multi-joint study with the second controller, the same two questions were presented to the participants again. Additionally, a final question assessed the subjective opinion comparing the two controllers:

3. I found one block more comfortable than the other.
(a) No
(b) Block 1
(c) Block 2.

\subsection{Data evaluation and statistics}

Statistical analysis was performed using the $\mathrm{R}$ environment for statistical computing (version 3.4.4., R Core Team, 2018). Linear mixed models were derived using "ImerTest" to assess the relationship between the primary outcomes ( $\tau_{\mathrm{m}, \mathrm{pHRI}}$ or $\tau_{\mathrm{p}, \mathrm{pHRI}}$ respectively) for the two speed levels and the two controllers. Since every axis is a different mechanical system, individual models were built to check significance at each axis. Since every subject had different anatomical and physiological parameters, a random effect for inter-subject variability and an additional random effect to account for different subjects and their interaction with the controller were introduced. Model assumptions were checked through visual examination of residual plots. The resulting mixed effect models used for both primary outcomes for each separate axis are:

$$
\begin{aligned}
\log \left(\tau_{\mathrm{m}, \mathrm{pHRI}}\right) \hat{=} \log \left(\tau_{\mathrm{p}, \mathrm{pHRI}}\right) \sim \text { Controller } * \text { Speed } \\
\\
+(1 \mid \text { Subject })+(1 \mid \text { Subject }: \text { Controller })
\end{aligned}
$$

A general linear hypothesis test with multiple comparisons for the linear mixed effect models was then performed using "glht()" of the R package "multcomp" to test for the significance and the effect of the controller on mean torque and mean peak torque for each axis-speed combination [20]. The function "ghlt()" adjusts the p-values by a single-step procedure, which incorporates correlations and is more powerful than the classical Bonferroni correction [20]. The significance level was set to (“*”, $p<0.05$ ). A

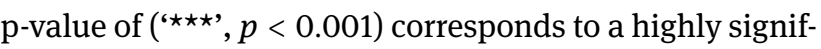
icant result.

The questionnaire statements 1) and 2) were analyzed with the Wilcoxon rank signed test using integer scores $(1, \ldots, 7)$ for the 7-point Likert scale. A simple percentage score was used to evaluate question 3). 
Single-Joint Transparency Study Results
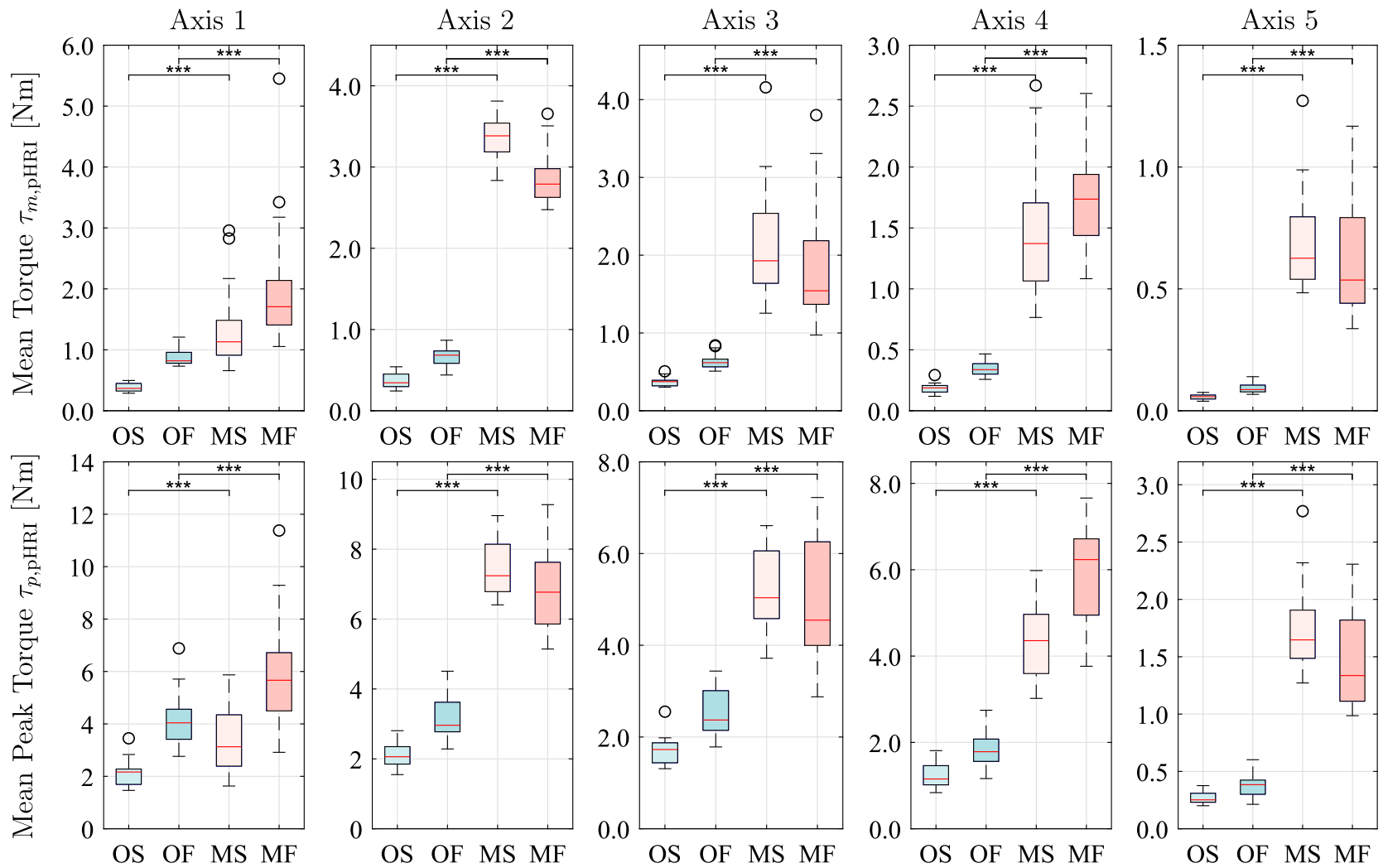

Observer Slow Speed (OS)

Observer Fast Speed (OF)

Model Slow Speed (MS)

Model Fast Speed (MF)

Figure 6: Single-joint transparency study: Mean absolute torque $\tau_{\mathrm{m}, \mathrm{pHRI}}$ and mean absolute peak torque $\tau_{\mathrm{p}, \mathrm{pHRI}}$ for all five tested axes of ARMin for the 20 subjects visualized as boxplots. The four scenarios are OS = Observer Slow Speed, OF = Observer Fast Speed, MS $=$ Model Slow Speed, MF = Model Fast Speed. All comparisons between disturbance observer and related feed-forward model scenario were highly significant (‘**”, $p<0.001)$.

\section{Results}

In this section the quantitative results from the single-joint and multi-joint transparency study are presented before the result from the questionnaire given to the subjects.

\subsection{Single-joint transparency study}

The disturbance observer "slow" speed scenario showed the lowest mean absolute interaction torque $\tau_{\mathrm{m}, \mathrm{pHRI}}$ for every of the five tested axes (see Fig. 6). The disturbance observer "fast" speed scenario showed the second lowest mean absolute interaction torques for every axis. Fig 6 illustrates that the velocity conditions in the model-based scenarios had a mixed effect on the mean absolute torque. The variability of the measured interaction torques as illustrated by the boxplot whisker range in Fig. 6 was lower with the disturbance observer by a factor of 2 to 10 compared to the model-based controller.
The mean peak torque $\tau_{\mathrm{p}, \mathrm{pHRI}}$ of the disturbance observer "slow" speed scenario resulted in the lowest values over all axes except for axis 1 (see Fig. 6). The disturbance observer "fast" speed scenario showed the second lowest mean torque peaks on every axis. The disturbance observer "fast" speed mean peak torque was higher than the "slow" speed mean peak torque on every axis. The mean peak torques for the feed-forward model-based scenario did not show velocity dependence.

\subsection{Multi-joint transparency study}

Similar to the single-joint transparency study, Fig. 7 shows that the disturbance observer "slow" speed scenario had the lowest mean torque and mean peak torque results over all axes. Additionally, the disturbance observer "fast" speed scenario had the second lowest mean torque and mean peak torque results of each axis. Overall, the disturbance observer scenarios showed highly significantly 


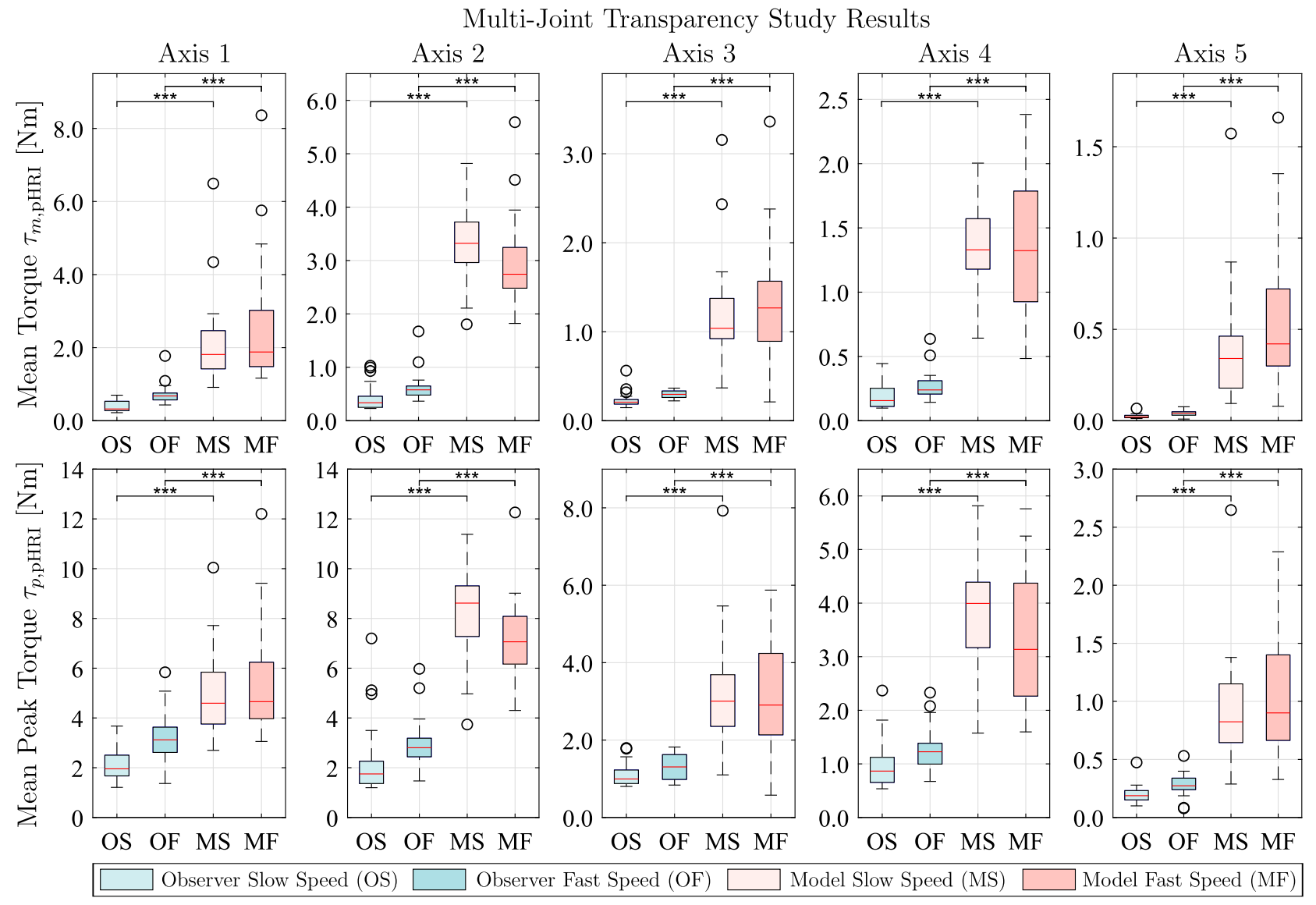

Figure 7: Multi-joint transparency study: Mean absolute torque $\tau_{\mathrm{m}, \mathrm{pHRI}}$ and mean absolute peak torque $\tau_{\mathrm{p}, \mathrm{pHRI}}$ for all five tested axes of ARMin for the 20 subjects visualized as boxplots. The four scenarios are OS = Observer Slow Speed, OF = Observer Fast Speed, MS = Model Slow Speed, MF = Model Fast Speed. All comparisons between disturbance observer and related feed-forward model-based scenario were highly significant (“***', $p<0.001$ ).

lower mean torque and mean peak torque values compared to the feed-forward model-based scenario counterparts (see Fig. 7). The effect of velocity in the feed-forward model measurements was axis-dependent and no overall systematic increase can be observed in the mean torque and mean peak torque plots. The variability of the measured interaction torques as illustrated by the boxplot whisker range in Fig. 7 was lower in the disturbance observer by a factor of 2 to 10 compared to the feed-forward model-based controller.

\subsection{Questionnaire}

The Wilcoxon test of question 1) revealed that people felt significantly less disturbances with the disturbance observer than with the feed-forward model-based controller $(p<0.001,95 \%$ confidence interval of $[1.5,3.0]$ points better). The evaluation of question 2) showed that exact movements were easier to perform with the disturbance observer compared to the feed-forward model-based controller $(p<0.001,95 \%$ confidence interval of $[2.0,3.5]$ points better). For question 3, $95 \%$ of the participants judged the disturbance observer to be more comfortable than the feed-forward model-based controller and 5\% (1 person) said that no differences were felt.

\section{Discussion}

In this section the different effects of the result section are discussed for the single-joint and multi-joint transparency study including the transfer of controller transparency characteristics between them, as well as the questionnaire.

\subsection{Single-joint transparency study}

The main goal of achieving higher transparency is reached by a reduction of mean torque and mean peak torque 
in axis movement direction. Fig. 6 shows clearly that the disturbance observer achieved significantly lower mean torques and mean peak torques compared to the feedforward model-based controller on every axis. Therefore, the disturbance observer achieved a significantly higher transparency than the feed-forward model-based controller for every axis and speed tested.

This is probably due to the fact that on one hand the disturbance observer is a feedback controller that reduces the system's error dynamics, while on the other hand the model-based controller is a feed-forward system that cannot react to the system's dynamics. Furthermore, for the model-based controller the missing inertial compensation supposably increased mean and mean peak torque values as evaluated in [18].

The systematic increase of disturbance observer mean torque and mean peak torque in Fig. 6 due to a velocity increase can be explained by the disturbance observer's low-pass filter limitations of the disturbance torque velocity as introduced in (12). This limitation was taken into account since ARMin only uses position sensors, so a double derivative to obtain acceleration data would be too noisy for control purposes.

The major boxplot whisker range differences between the controllers indicate that the disturbance observer has a much lower inter-subject variability compared to the feedforward model-based controller. Subsequently, high level control can be reliably tuned for further studies and effects from old studies on the ARMin with the disturbance observer could be more significant now.

\subsection{Multi-joint transparency study}

Due to similar and clear results as in the single-joint study, the same advantages of the disturbance observer over the model-based controller apply for multi-joint movements as for single-joint movements. Furthermore, the main statement of the multi-joint study is the direct transfer of the assessed benefits of the disturbance observer to a complex and multidimensional movement. This successful transfer is probably mainly due to decoupling of the upper and lower arm rotational axes (axis 3 and axis 5) in the Jacobian leading to more similar single-joint control in multi-joint movements. Another main reason is supposably the applied characteristic of the disturbance observer trying to remove all non-diagonal entries in the mass matrix $\boldsymbol{M}_{\text {nom }}$ as defined in (7). Since the model-based feedforward controller did entail neither particular characteristics, no distinct transfer similarities from single-joint to multi-joint evaluation could be observed. Even with the discussed differences of axes level transparency evalua- tion and sensor level transparency evaluation, it is expected that also the force/torque transparency evaluation on the sensor level [12] would have lead to clear results in favor of the disturbance observer. The tested movement covered activities of daily living for our patients. Therefore, the transparency study on multi-joint movements showed a representative transfer of the results to the clinical application of the multi-axis rehabilitation robot ARMin IV+.

\subsection{Questionnaire}

The subjective rating of the participants in all questions significantly supported the quantitative data presented. Overall, $95 \%$ of the participants preferred the disturbance observer and found it more comfortable, thus demonstrating that the assessed technical reliability also transfers to the subjective perception of the participants.

\section{Conclusion}

In this paper, a systematic method for velocity-dependent transparency evaluation on an axis level is presented that can be expanded to the multi-joint level for generalization. The transparency evaluation was consequently applied on the currently used feed-forward model-based and a disturbance observer for the arm exoskeleton robot ARMin IV+.

In comparison, the disturbance observer significantly outperformed the model-based feed-forward controller in transparency, reliability, and systematic performance for every test speed and axis. The transfer to multi-axis movements was shown to be straightforward with similar highly significant results probably due to Jacobian and disturbance observer characteristics of partial axes decoupling. The significant questionnaire results strengthened the quantitative data. Most importantly, the disturbance observer is available in form of a general framework that only requires adaption of a few parameters, while modelbased controllers depend on the quality of the model, require models for different effects, and do not necessarily show consistent behavior across working range and velocity range.

Due to its evident advantages, the disturbance observer will be employed in ARMin devices as a standard controller in future research at the Sensory-Motor-System Lab of ETH Zurich. It is expected that positive clinical results from previous work with ARMin [1] still hold and the use of the disturbance observer as used transparency controller would have led to even clearer results in favor of robot-assisted rehabilitation with ARMin. 
Acknowledgment: The authors would like to thank Michael Herold-Nadig and Yves Zimmermann for their constant, valuable support.

Funding: This work was supported by ETH research grant 0-20075-15, ETH, UZH and the CRRP Neuro-Rehab, University of Zurich.

\section{References}

1. V. Klamroth-Marganska, J. Blanco, K. Campen, A. Curt, V. Dietz, T. Ettlin, M. Felder, B. Fellinghauer, M. Guidali, A. Kollmar and et al., Three-dimensional, task-specific robot therapy of the arm after stroke: a multicentre, parallel-group randomised trial, The Lancet Neurology 13(2) (2014), 159-166.

2. G. Kwakkel, B. J. Kollen and H. I. Krebs, Effects of robot-assisted therapy on upper limb recovery after stroke: a systematic review, Neurorehabilitation and neural repair 22(2) (2008), 111-121.

3. J. Mehrholz, M. Pohl, T. Platz, J. Kugler and B. Elsner, Electromechanical and robot-assisted arm training for improving activities of daily living, arm function, and arm muscle strength after stroke, The Cochrane Library (2015).

4. N. Jarrassé, T. Proietti, V. Crocher, J. Robertson, A. Sahbani, G. Morel and A. Roby-Brami, Robotic exoskeletons: a perspective for the rehabilitation of arm coordination in stroke patients, Frontiers in human neuroscience 8 (2014), 947.

5. T. Proietti, V. Crocher, A. Roby-Brami and N. Jarrassé, Upper-limb robotic exoskeletons for neurorehabilitation: a review on control strategies, IEEE reviews in biomedical engineering 9 (2016), 4-14.

6. G. Rosati, R. Secoli, D. Zanotto, A. Rossi and G. Boschetti, Planar robotic systems for upper-limb post-stroke rehabilitation, in: ASME 2008 International Mechanical Engineering Congress and Exposition, American Society of Mechanical Engineers, pp.115-124, 2008.

7. H. Kim, L. M. Miller, Z. Li, J. R. Roldan and J. Rosen, Admittance control of an upper limb exoskeleton-Reduction of energy exchange, in: Engineering in Medicine and Biology Society (EMBC), 2012 Annual International Conference of the IEEE, IEEE, pp. 6467-6470, 2012.

8. M. Bannwart, M. Bolliger, P. Lutz, M. Gantner and G. Rauter, Systematic analysis of transparency in the gait rehabilitation device the FLOAT, in: Control, Automation, Robotics and Vision (ICARCV), 2016 14th International Conference on, IEEE, pp. 1-6, 2016.

9. H. Vallery, A. Duschau-Wicke and R. Riener, Optimized passive dynamics improve transparency of haptic devices, in: Robotics and Automation, 2009. ICRA'09. IEEE International Conference on, IEEE, pp. 301-306, 2009.

10. W. van Dijk, H. van der Kooij, B. Koopman and E. H. F. Van Asseldonk, Improving the transparency of a rehabilitation robot by exploiting the cyclic behaviour of walking, in: Rehabilitation Robotics (ICORR), 2013 IEEE International Conference on, IEEE, pp. 1-8, 2013.

11. R. Vertechy, A. Frisoli, A. Dettori, M. Solazzi and M. Bergamasco, Development of a new exoskeleton for upper limb rehabilitation, in: Rehabilitation Robotics, 2009. ICORR 2009. IEEE International Conference on, IEEE, pp. 188-193, 2009.

12. N. Jarrasse, M. Tagliabue, J. V. G. Robertson, A. Maiza, V. Crocher, A. Roby-Brami and G. Morel, A methodology to quantify alterations in human upper limb movement during co-manipulation with an exoskeleton, IEEE Transactions on neural systems and Rehabilitation Engineering 18(4) (2010), 389-397.

13. J. F. Veneman, R. Ekkelenkamp, R. Kruidhof, F. C. T. van der Helm and $H$. van der Kooij, A series elastic-and bowden-cable-based actuation system for use as torque actuator in exoskeleton-type robots, The international journal of robotics research 25(3) (2006), 261-281.

14. P. E. Dupont, Avoiding stick-slip through PD control, IEEE Transactions on automatic control 39(5) (1994), 1094-1097.

15. A. Sabanovic and K. Ohnishi, Motion control systems, John Wiley \& Sons, 2011.

16. D. A. Lawrence, Stability and transparency in bilateral teleoperation, IEEE transactions on robotics and automation 9(5) (1993), 624-637.

17. F. Just, K. Baur, R. Riener, V. Klamroth-Marganska and G. Rauter, Online adaptive compensation of the ARMin Rehabilitation Robot, in: Biomedical Robotics and Biomechatronics (BioRob), 2016 6th IEEE International Conference on, IEEE, pp. 747-752, 2016.

18. F. Just, K. Baur, V. Klamroth-Marganska, R. Riener and G. Rauter, Motor Inertia Compensation of the ARMin Rehabilitation Robot, in: AUTOMED 2016: Workshop Wismar, 22.-23. September 2016 / Deutsche Gesellschaft für Biomedizinische Technik im VDE (0. Simanski, O. Hagendorf and J. Zucknik, eds.), Hochschule Wismar, 2016 (en).

19. K. R. S. Holzbaur, W. M. Murray and S. L. Delp, A model of the upper extremity for simulating musculoskeletal surgery and analyzing neuromuscular control, Annals of biomedical engineering 33(6) (2005), 829-840.

20. T. Hothorn, F. Bretz and P. Westfall, Simultaneous Inference in General Parametric Models, Biometrical Journal 50(3) (2008), 346-363.

\section{Bionotes}

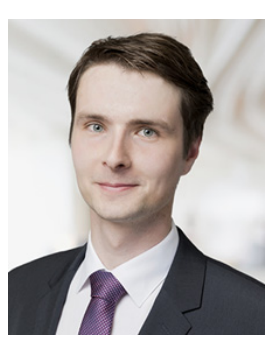

Fabian Just

Sensory-Motor Systems Lab, ETH Zurich and Spinal Cord Injury Center, University Hospital Balgrist, Zurich, Switzerland fabian.just@hest.ethz.ch

Fabian Just received a M.Sc. degree in electrical and computer engineering from Purdue University (IN, USA) as well as a M.Sc. degree in automatic control from Ruhr-University Bochum (Germany). He is a PhD student at the Sensory-Motor Systems Lab of ETH Zurich. His main research focus is robotics, automatic control, machine learning, and neurorehabilitation. 


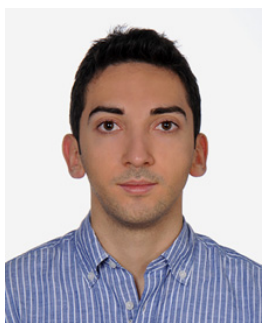

\section{Özhan Özen}

ARTORG Center for Biomedical Engineering Research, Gerontechnology \& Rehabilitation, University of Bern, Bern, Switzerland oezhan.oezen@artorg.unibe.ch

Özhan Özen received his B.S. in Mechatronics Engineering from Sa banci University, and his MSc in Robotics, Systems \& Control from ETH Zurich. He started his PhD at University of Bern, under the supervision of Laura Marchal-Crespo in 2017. His focus is making the robotics systems intelligent, adaptive and autonomous for neurorehabilitation.

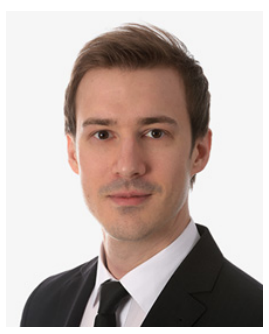

\section{Philipp Bösch}

Sensory-Motor Systems Lab, ETH Zurich and Spinal Cord Injury Center, University Hospital Balgrist, Zurich, Switzerland phboesch@student.ethz.ch

Philipp Bösch received his B.Sc. in health science and technology from the ETH Zurich in 2017. He is currently finishing his M.Sc. in health science and technology focusing on medical technologies and rehabilitation engineering.

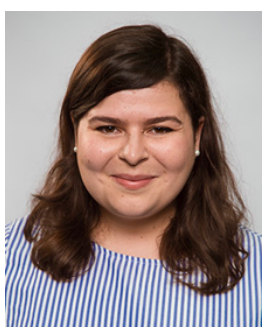

\section{Hanna Bobrovsky}

Sensory-Motor Systems Lab, ETH Zurich and Spinal Cord Injury Center, University Hospital Balgrist, Zurich, Switzerland hannab@student.ethz.ch

Hanna Bobrovsky is a Master's student majoring in Medical Technology at the ETH Zurich. Her current research interests involve neurorehabilitation, rehabilitation robotics and prosthetics.

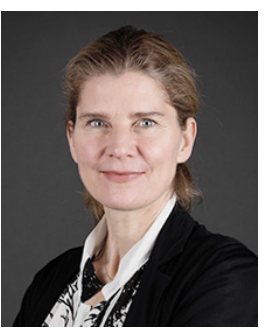

Verena Klamroth-Marganska

Sensory-Motor Systems Lab, ETH Zurich and Spinal Cord Injury Center, University Hospital Balgrist, Zurich, Switzerland verena.klamroth@hest.ethz.ch
Verena Klamroth studied Human Medicine at Freie Universität and Humboldt-Universität in Berlin (Germany). She received her doctoral degree from Westfälische Wilhelms-Universität Münster (Germany). Since 2008, she has been a medical advisor and the group leader of the ARMin project at the Sensory-Motor Systems Lab at ETH Zurich.

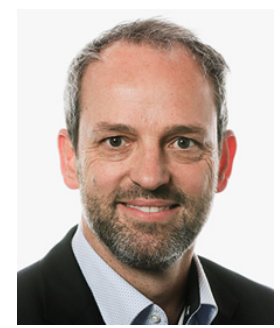

\section{Robert Riener}

Sensory-Motor Systems Lab, ETH Zurich and Spinal Cord Injury Center, University Hospital Balgrist, Zurich, Switzerland robert.riener@hest.ethz.ch

Robert Riener is full professor for Sensory-Motor Systems at the Department of Health Sciences and Technology, ETH Zurich, and full professor of medicine at the University Hospital Balgrist, University of Zurich. He obtained a MSc in mechanical engineering in 1993 and a PhD in biomedical engineering 1997, both from TU München, Germany. In 2003 he became professor in Zurich. His main research focus is in rehabilitation robotics, virtual reality, and biomechanics. Riener has published more than 400 peer-reviewed articles, 20 book chapters and filed 23 patents. He is the initiator and organizer of the Cybathlon.

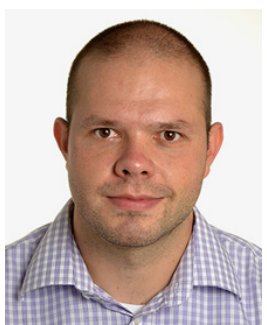

\section{Georg Rauter}

Sensory-Motor Systems Lab, ETH Zurich and Spinal Cord Injury Center, University Hospital Balgrist, Zurich, Switzerland BIROMED-Lab, DBE, University of Basel, Basel, Switzerland georg.rauter@unibas.ch

Georg Rauter is assistant professor for medical robotics and mechatronics at the Department of Biomedical Engineering of University of Basel. The main focus of the research in his BIROMED Lab is: robotic endoscopes for laser ablation of hard tissue, bio-inspired sensor technologies for endoscopic navigation, tele manipulation, automation, kinematics, rehabilitation robotics, and control. 